OPEN@ACCESS

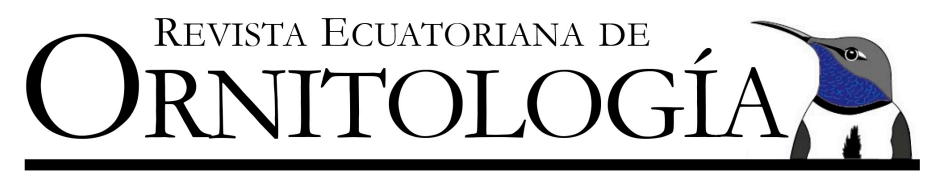

COMUNICACIÓN CORTA/SHORT COMMUNICATION

\title{
El Caracara Montañero Phalcoboenus megalopterus (Falconiformes: Falconidae), registro de una nueva localidad al occidente de la provincia de Azuay
}

\author{
Santiago Barros*, Boris Landázuri, Eduardo A. Barnuevo, Pedro X. Astudillo \\ Escuela de Biología, Universidad del Azuay, Av. 24 de mayo 7-77 y Hernán Malo, Cuenca, Ecuador. \\ *Autor para correspondencia/Corresponding author, e-mail: jsanty.b1@gmail.com \\ Editado por/Edited by: Juan Freile \\ Recibido/Received: 4 Junio 2019 Aceptado/Accepted: 18 Noviembre 2019 \\ Publicado en línea/Published online: 22 Marzo 2020
}

\section{Mountain Caracara Phalcoboenus megalopterus (Falconiformes: Falconidae), a new locality in western Azuay Province}

\section{Resumen}

En Ecuador, el Caracara Montañero Phalcoboenus megalopterus se distribuye en los Andes del extremo sur. Los registros mayoritariamente se concentran en las provincias de Loja y Zamora Chinchipe. Sin embargo, al norte del límite conocido, en la provincia de Azuay, los registros de P. megalopterus son escasos y sin documentación. En esta nota se presenta el primer registro fotográfico en la provincia de Azuay. Un individuo adulto fue observado en agosto de 2018 en San Gerardo, cantón Girón, en los Andes occidentales de la provincia. Este registro representa una nueva localidad, las más septentrional, y sugiere que la especie podría distribuirse localmente hacia el norte del límite de su distribución conocido en Ecuador.

Palabras clave: Andes, bosque altoandino, Girón, distribución, Ecuador, Phalcoboenus megalopterus.

\begin{abstract}
In Ecuador, the Mountain Caracara Phalcoboenus megalopterus ranges in the southernmost Andes. Records come mainly from the provinces of Loja and Zamora Chinchipe. However, there are a few visual records north of the known distributional limit, in the province of Azuay, all undocumented. We present the first photographic record for Azuay Province. In August 2018, we observed and photographed one adult at San Gerardo, canton Girón, western Andes of Azuay. This record represents a new, northernmost locality, and suggests that the species may be locally distributed north of its current known distribution.
\end{abstract}

Keywords: Andes, Andean montane forest, Girón, distribution, Ecuador, Phalcoboenus megalopterus.

El Caracara Montañero Phalcoboenus megalopterus se distribuye principalmente en regiones andinas (>3500 m s.n.m.) de Perú, Bolivia, Chile y Argentina. En Ecuador ocupa la región altoandina al sur del país, en las provincias de Zamora Chinchipe y Loja (Fjeldså \& Krabbe, 1990; Bierregaard \& Kirwan, 2019). Esta especie ocupa predominantemente el ecosistema de páramo entre 2900-3800 m s.n.m. (McMullan \& Navarrete, 2013; Freile \& Poveda, 2019) (Fig 1). Es posible encontrarla en áreas abiertas forrajeando sola o en parejas.

Esta especie puede ser confundida con el Caracara Curiquingue $P$. carunculatus (Ridgely \& Greenfield, 2001), especie endémica de los páramos andinos centrales (Stattersfield et al., 1998), que en Ecuador se distribuye desde la provincia de Azuay hacia el norte (Fjeldså \& Krabbe, 1990; Freile \& Poveda, 2019). La zona limítrofe entre Loja y Azuay se ha sugerido como posible zona de contacto entre P. megalopterus y P. carunculatus (Poulsen, 1993), lo cual sugiere una estrecha zona de simpatría entre estas dos especies (Ridgely \& Greenfield, 2001).

El 30 de agosto de 2018 se fotografió un individuo adulto (Fig. 2) perchado por $c .15$ min en la copa de un pino (Pinus patula) $(-3,140737,-79,186678,2839$ m s.n.m). El hábitat era un mosaico de cultivos con remanentes de bosque montano en San Gerardo, cantón Girón, Andes suroccidentales de Azuay (Fig. 1). El individuo fue 
identificado por su plumaje: garganta inferior y pecho negro entero sin listas blancas, rostro con una ligera proporción de piel roja implume y escasa detrás del ojo (Ridgely \& Greenfield, 2006) (Fig. 2).

En Ecuador existen numerosos reportes de P. megalopterus, pero solamente 13 registros reportados en eBird disponen de evidencia fotográfica, todos ellos en la provincia de Loja (e.g., Davies, 2010; Miller, 2017; Ahlman, 2018; Vanegas, 2018). En la provincia de Azuay existen tres registros recientes: 1) Llaviucu, $16 \mathrm{~km}$ al noroeste de Cuenca; 2) cerca de la parroquia Jima, 29 km al sur de Cuenca; y 3) La Ramada, 43 km al sur de Cuenca (Black, 2015; Caddy, 2018; Licharson, 2019). No obstante, estos registros no presentan evidencia fotográfica u otro tipo de documentación, por lo que no es posible verificar si la identificación es acertada. Esto pone en evidencia la necesidad de documentar las observaciones de $P$. megalopterus fuera de su área regular de distribución.

Este reporte constituye el primer registro fotográfico de P. megalopterus en la provincia de Azuay, al norte del límite conocido de distribución de la especie. Los registros documentados más próximos son en Cerro de Arcos, c. $57 \mathrm{~km}$ al suroeste, provincia de Loja (Ahlman; 2018; Vanegas; 2018). Esta nueva localidad sugiere que su área de distribución podría ampliarse hacia el norte, con posibles registros localizados en los Andes del centrosur de Ecuador. El registro de $P$. megalopterus dentro del rango de distribución conocido de $P$. carunculatus y los registros de $P$. carunculatus reportados por Poulsen (1993) dentro del rango de distribución conocido de $P$. megalopterus podrían sugerir que la zona de contacto entre estas dos especies es más amplia y se extiende más hacia el norte de lo reportado anteriormente (Poulsen, 1993). Es necesario desarrollar más estudios para comprender la distribución y las relaciones ecológicas entre estas dos especies en la aparente zona de contacto.

La identificación de campo entre $P$. megalopterus y $P$. carunculatus (los juveniles son casi indistinguibles; Ridgely \& Greenfield, 2001, 2006) podría dificultar la determinación de los límites de distribución y zona de contacto de estas especies, y generar información errónea sobre nuevos registros para ambas especies. Por lo tanto, los registros al norte del área de distribución conocida de P. megalopterus deben ser sustentados con evidencia.

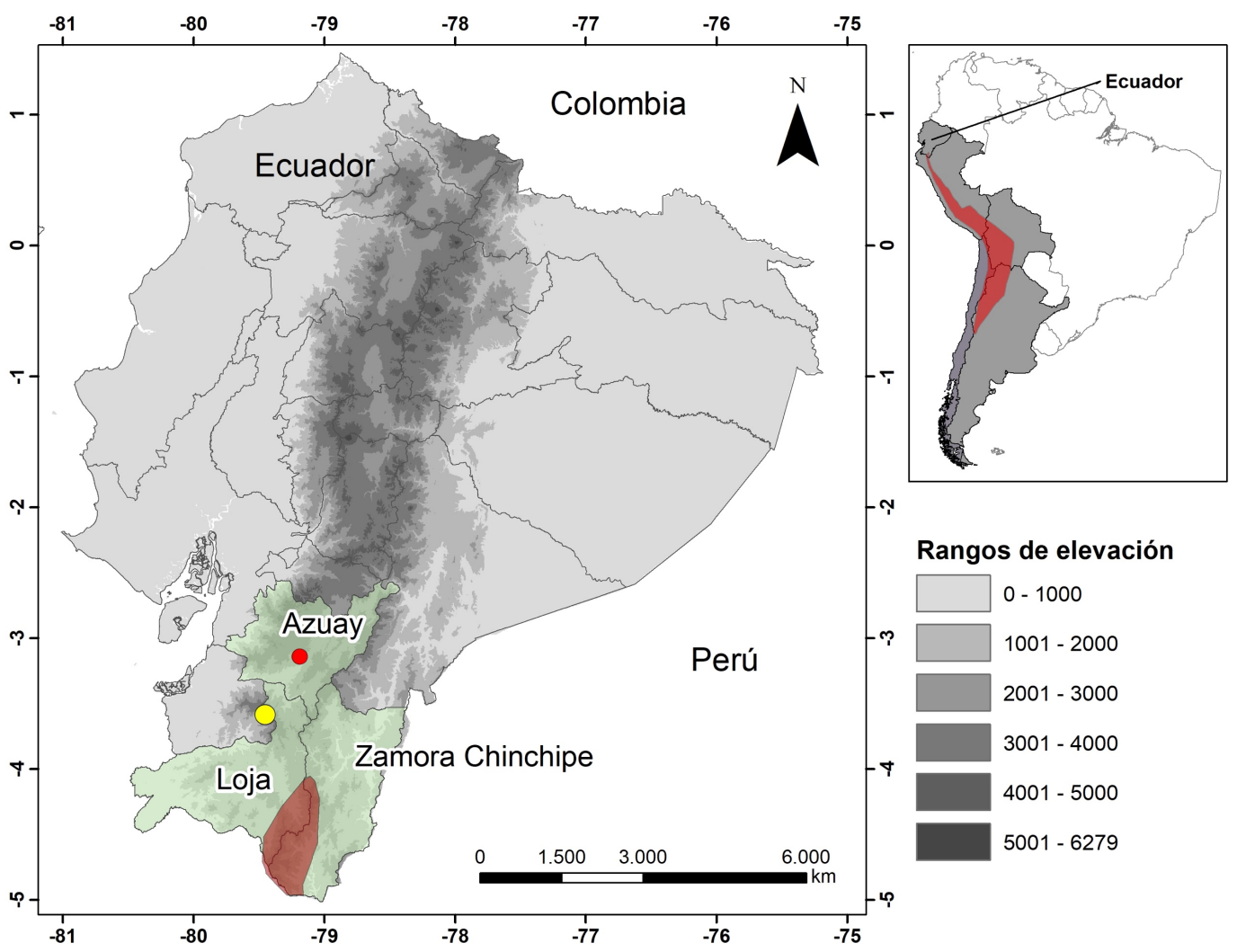

Figura 1: Nueva localidad del Caracara Montañero Phalcoboenus megalopterus en Ecuador (círculo rojo); registro documentado más cercano (Cerro de Arcos, provincia de Loja) (círculo amarillo); área de distribución de la especie en Sudamérica y Ecuador (polígono rojo; BirdLife International, 2016). 


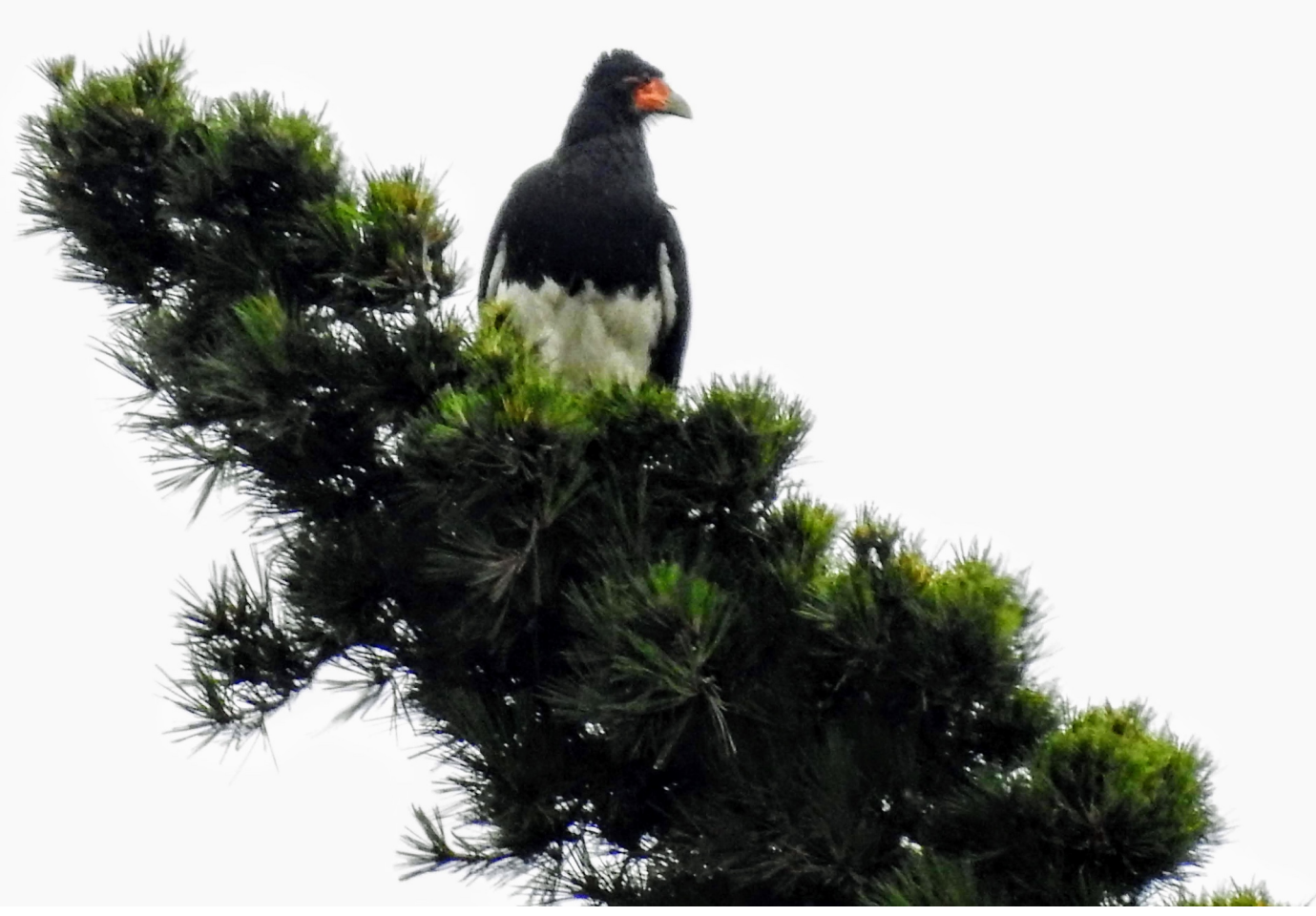

Figura 2: Caracara Montañero Phalcoboenus megalopterus en San Gerardo, cantón Girón, provincia del Azuay, 30 de Agosto de 2018 (Boris Landázuri).

\section{AGRADECIMIENTOS}

Este estudio fue financiado por la Universidad del Azuay (Fondos UDA: 2018-0088) y por INVMETALS. Los permisos de investigación fueron otorgados por el Ministerio del Ambiente de Ecuador (Licencia: No. 1832018-DPAA/MA). Nuestro agradecimiento a Vicente Jaramillo y Jorge Barreno de INVMETALS Ecuador por su apoyo constante a nuestras investigaciones. Agradecemos también a Jacinto Guillén, Francisco Salgado, David Siddons, Antonio Crespo y Andrés López de la Universidad del Azuay.

\section{REFERENCIAS}

Ahlman, R. (2018, Junio 13). eBird checklist https://ebird.org/checklist/S53054898. eBird: an online database of bird distribution and abundance. Ithaca, New York. URL: http://www.ebird.org.

Bierregaard, R.O. \& Kirwan, G.M. (2019). Mountain Caracara (Phalcoboenus megalopterus). In: del Hoyo, J., Elliott, A., Sargatal, J., Christie, D.A. \& de Juana, E. (eds.). Handbook of the birds of the world alive. Barcelona, España: Lynx Edicions. URL: https://www.hbw.com/node/53190.

Black, K. (2015, Febrero 11). eBird checklist https://ebird.org/checklist/S23053779. eBird: an online database of bird distribution and abundance. Ithaca, New York. URL: http://www.ebird.org.

BirdLife International. (2016). Species factsheet: Phalcoboenus megalopterus. Cambridge, Reino Unido: BirdLife International. URL: http://www.birdlife.org/datazone/. 
Caddy, J. (2018, Febrero 28). eBird checklist https://ebird.org/checklist/S43510881. eBird: an online database of bird distribution and abundance. Ithaca, New York. URL: http://www.ebird.org.

Davies, I. (2010, Diciembre 31). eBird checklist: https:/ebird.org/checklist/S7344321. eBird: an online database of bird distribution and abundance. Ithaca, New York. URL: http://www.ebird.org.

Fjeldså, J., \& Krabbe, N. (1990). Birds of the high Andes. Svendborg \& Copenhagen, Dinamarca: University of Copenhagen \& Apollo Books.

Freile, J.F. \& Poveda, C. (2019, Mayo 21). Phalcoboenus megalopterus. En: Freile, J.F. \& Poveda, C. (Eds.), Aves del Ecuador. Versión 2019.0. Quito, Ecuador: Museo de Zoología, Pontificia Universidad Católica del Ecuador. URL: https://bioweb.bio/faunaweb/avesweb/FichaEspecie/Phalcoboenus\%20megalopterus.

Licharson, J. (2019, Enero 19). eBird checklist https://ebird.org/checklist/S51835509. eBird: an online database of bird distribution and abundance. Ithaca, New York. URL: http://www.ebird.org.

McMullan, M. \& Navarrete, L. (2013). Fieldbook of the birds of Ecuador, including the Galápagos Islands. Quito, Ecuador: Fundación de Conservación Jocotoco.

Miller, B. (2017, Octubre 26). eBird checklist https://ebird .org/checklist/S40197851. eBird: an online database of bird distribution and abundance. Ithaca, New York. URL: http://www.ebird.org.

Poulsen, B.O. (1993). A contact zone between Mountain and Carunculated Caracaras in Ecuador. Wilson Bulletin, 105, 688-691. URL: http://www.jstor.org/stable/4163362.

Ridgely, R.S., \& Greenfield, P.J. (2001). The birds of Ecuador. Field guide. Ithaca, NY: Cornell University Press.

Ridgely, R.S., \& Greenfield, P.J. (2006). Aves del Ecuador: guía de campo. Quito, Ecuador: Academia de Ciencias Naturales de Filadelfia \& Fundación de Conservación Jocotoco.

Stattersfield, A.J., Crosby, M.J., Long, A.J. \& Wege, D.C. (1998). Endemic bird areas of the world. Priorities for biodiversity conservation. Cambridge, Reino Unido: BirdLife International Conservation Series 7.

Vanegas, A. (2018, Diciembre 15). eBird checklist https://ebird.org/checklist/S51211774. eBird: an online database of bird distribution and abundance. Ithaca, New York. URL: http://www.ebird.org. 